\title{
Environmental aspects of education: assessment of adaptation of students with different types of autonomic regulation during physical exertion
}

\author{
Victor Gorelik ${ }^{1,2, *}$, Valery Vlasov $^{1}$, Alyona Podlubnaya ${ }^{1}$, and Andrey Piyanzin ${ }^{1}$ \\ ${ }^{1}$ Togliatti State University, Department of Adaptive Physical Education, Sports and Tourism, 445020, \\ Samara Region, Togliatti, Russian Federation \\ ${ }^{2}$ Russian State Social University, Faculty of Physical Education, 129226, Moscow, Russian \\ Federation
}

\begin{abstract}
The environmental aspects of education include the study of the processes of adaptation of students to the effects of the school environment and increasing teaching loads. This problem most often remains an important problem of age physiology and school hygiene. However, its meaning is much broader. Modern studies of the adaptation of students to the effects of the school environment and increasing educational loads have intensified in connection with the tasks of transforming education in the direction of its ecological culture. Our study proceeds from the assumption that the possibilities of assessing the environmental friendliness or environmental culture of education may be associated with the development of an instrumental method for determining and analyzing heart rate variability, suitable for examining large contingents of students as part of such methods for assessing the environmental friendliness of education and its other characteristics as a screening method and monitoring. Evaluation of heart rate variability allows determining the state of the mechanisms of regulation of physiological functions of the human body, the activity of regulatory systems: the central nervous system, the autonomic nervous system, intracardiac processes of the heart, the balance of the sympathetic and parasympathetic divisions of the autonomic nervous system.
\end{abstract}

\section{Introduction}

The environmental aspects of education include the study of the processes of adaptation of students to the effects of the school environment and increasing teaching loads. This problem most often remains an important problem of age physiology and school hygiene [1-2]. However, its meaning is much broader. Modern studies of the adaptation of students to the effects of the school environment and increasing educational loads have intensified in connection with the tasks of transforming education in the direction of its ecological

*Corresponding author: lecgoy@list.ru 
culture. Numerous scientific studies and life experience of a person indicate that regular exercise has a healing effect on all systems and functions of the human body, provide it with the necessary charge of vigor and good mood. Before everyone who decides to join physical exercises, the question arises: what type of exercise is most effective? One of the most important management groups are those that activate and harmonize the work of the cardiovascular system. In modern conditions, it is the cardiovascular system that turns out to be the weakest link in our body. There are many ways to strengthen it, for example, jogging. He gained immense popularity due to its effectiveness, general availability, naturalness. But no matter which of the running programs a person chooses, he cannot do without gymnastics. Gymnastics has a general physiological effect on the body, increasing metabolism, has a positive effect on the cardiovascular, respiratory and digestive systems, develops strength, flexibility, speed, endurance, and coordination of movements.

Our study proceeds from the assumption that the possibilities of assessing the environmental friendliness or environmental culture of education may be associated with the development of an instrumental method for determining and analyzing heart rate variability, suitable for examining large contingents of students as part of such methods for assessing the environmental friendliness of education and its other characteristics as a screening method and monitoring [3-4]. Evaluation of heart rate variability allows to determine the state of the mechanisms of regulation of physiological functions of the human body, the activity of regulatory systems: the central nervous system (CNS), the autonomic nervous system (ANS), intracardiac processes of the heart, as well as the balance of the sympathetic and parasympathetic divisions of the autonomic nervous system (ANS) [5-7].

\section{Materials and methods}

The study took place in the city of Togliatti, Samara region of Russia from October 2019 to April 2020 on the basis of school number 90 and was divided into three stages.

At the first stage (October 2019), scientific and methodological literature on the problem of this research was studied, as a result of which the tasks were set and the research methods were determined.

At the second stage (April 2020), an experiment was conducted to examine students in the experimental group (EG) and the control group (CG). The study involved 20 boys aged 12-13 years. The experimental group and the control group consisted of 20 boys in each group. Pupils from the EG and CG were engaged in physical culture three times a week. The EG included exercises for children with different TVR, and the children in the CG were engaged in a standard physical culture program.

At the third stage (October 2020), repeated testing was carried out in order to determine the influence of the developed optimizing methodology of physical culture lessons in the EG and the CG.

To assess the differences in the level of the studied trait at the beginning and at the end of the experiment in the EG and CG, the Student's t-test was used.

Evaluation of cardiointervalograms and analysis of heart rate variability (HRV) was carried out using the hardware-software complex "Varicard 2.51" [8].

In assessing the group differences in the study, the following indicators were taken into account: TBR (or type of autonomic regulation), TP (total power of the HRV spectrum, $\mathrm{ms}^{2}$ ), HR (heart rate, beats per minute), RR (average duration of intervals between cardiac contractions, ms), MxDMn (difference between maximum and minimum values of cardio intervals), RMSSD (indicator of activity of the parasympathetic link of autonomic regulation, ms), Amo50\% / 50 (indicator activity of the sympathetic link of regulation, ms), $\mathrm{Si}$ (stress index or index of tension of regulatory systems in arbitrary units). 


\section{Results}

Optimal (striking a person, harmonizing, allowing coping with difficulties and stresses) physical activity contributes to the expansion of the body's reserve capabilities and resistance to the effects of various unfavorable factors. Physical education as a subject of higher education is not only a means of health promotion, but also an integral part of education and professional training of the students, education of the need for systematic physical exercises. An important aspect of the health-improving effect of physical exercises is the regulatory-trophic effect of their use. The action of physical exercises is to activate the processes of tissue nutrition, to stimulate and normalize metabolism, improve regeneration processes, and increase blood flow to tissues.

Fatigue can be characterized as a reversible violation of physiological and biochemical homeostasis, which is compensated for in the post-work recovery period. The causes of fatigue are diverse and there is no single theory of the problem, since work processes or various types of physical exercises leading to fatigue are too different in structure, tension and character. The degree of functional changes in the body during fatigue and the speed of recovery during the rest period depend on the fitness of the person. If the untrained period of rest is accompanied by disturbances in functional shifts, then in the trained one this goes away with moderate functional shifts and a feeling of pleasant fatigue.

Functional overload

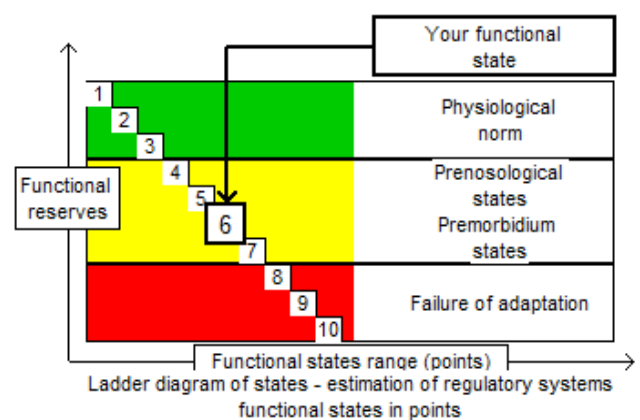

Fig. 1. Assessment of the functional state and regulatory systems of students at the beginning of the EG study

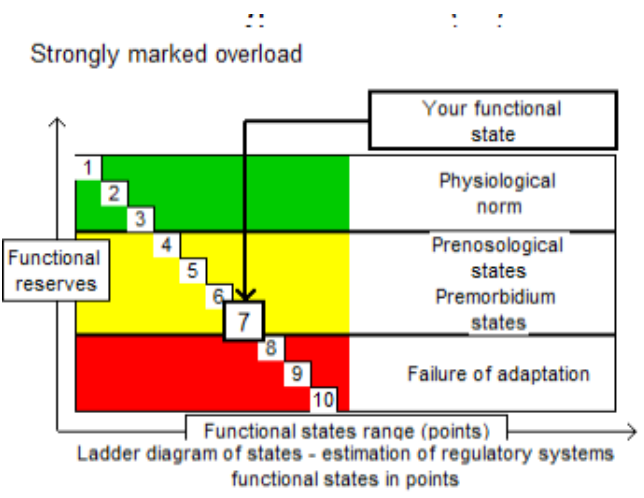

Fig. 2. Assessment of the functional state and regulatory systems of students at the beginning of the study CG

The health-improving role of physical exercise is associated with the stimulating effect of fatigue as a stimulus for recovery processes. If the body loses fatigue, then the recovery 
processes decrease, as well as the tone of the nervous system. The correct dosage of the load, the gradual increase in its volume and the possession of relaxation (relaxation) skills are the basis for neutralizing the overstrain of the body. Systematic, planned, balanced and directed use of (complex) physical exercises in different modes of educational and labor activities of students will contribute to the development of functional adaptation of the body to physical stress, including unfavorable factors (correction of mental stress, alignment of other disturbed body functions).

The presence of deviations of the functional character in the work of the cardiovascular system in the EG and CG led to the need to use a differentiated approach at physical culture lessons, taking into account the state of tension of the regulatory systems of students and the selection of the appropriate motor activity, which contributes to an increase in the functional state of the cardiovascular system and the health-improving orientation of physical training culture [9, 10] (Figures 1,2).

Comparative analysis of heart rate variability indicators in children of the second childhood period with different types of autonomic regulation of types I, II, III and IV established significant differences between the indicators.

So, according to the presented data, significant differences were determined for children with the first type of autonomic regulation with a moderate predominance of central regulation of the heart rate in the EG and CG groups according to the following indicators: HR (bpm or beats per minute), RR (ms), MxDMn (ms), Amo50\%/50 (ms), Si (conventional units). In the presence of reliable results, it is important to note that in the EG there are more optimal values of HRV indices in comparison with the CG. This indicates the effectiveness of the use of health-improving programs in the EG (Table 1).

In children of the second childhood with type II autonomic regulation with a characteristic pronounced predominance of central regulation, the following reliable values were obtained for the following HRV indicators: HR (beats per minute), RR (ms), RMSSD (ms), Amo50\%/50 (ms), Si (conventional units), TP $\left(\mathrm{ms}^{2}\right)$. The obtained values of indicators in independent samples of the studied groups EG and $\mathrm{CG}$ and their changes are reliable: the results described by us are reliable; the method of assessing changes was applied correctly. This can also be interpreted as a positive impact of using specific physical exercises and effective physical education. In the comparative analysis, it is important to note a decrease in HRV indices, and, as a consequence, release of tension in the regulatory systems of the body in the EG in comparison with the CG (Table 1).

In a comparative analysis in children with the third type of autonomic regulation with a characteristic moderate predominance of autonomic regulation of the heart rate, the following reliable HRV indicators were obtained: HR (beats per minute), RR (ms), MxDMn (ms), RMSSD (ms), Amo50\% / 50 (ms), Si (conventional units), TP (ms ${ }^{2}$ ). In the considered groups EG and CG reliable values were established between all considered HRV indices. Children have a significantly longer R-R duration (ms) and a spread of MxDMn cardio intervals, much more RMSSD value, less Si, a moderate total power TP. This indicates the most optimal interaction between the sympathetic and parasympathetic divisions of the ANS (Table 1).

When analyzing HRV indicators in children with type IV autonomic regulation with a characteristic with a pronounced predominance of autonomous regulation, reliable values were obtained for the following indicators: heart rate (bpm), RR (ms), MxDMn (ms), RMSSD (ms ), TP $\left(\mathrm{ms}^{2}\right)$. Children with type IV autonomic regulation are characterized by the largest R-R values of the duration of cardio intervals, MxDMn (ms) spread of cardio intervals, the lowest values of Amo50\% / 50 (ms), Si, (conventional units). In the comparative analysis it was found that in the EG the values of HRV are the most optimal in comparison with the CG, there is a smaller scatter of the considered indicators, which confirms the correctness of the use of correctional-developmental exercises. Significant 
high values of HRV indices in children with type IV autonomic regulation can be considered as imperfection, underdevelopment of regulation systems in ontogenesis or as a marker that determines an individual approach to children with type IV autonomic regulation using specially designed programs (Table 1 ).

Table 1. HRV indices in children of the second childhood period at the end of the EG and CG study $(\mathrm{M}+\mathrm{m})$

\begin{tabular}{|c|c|c|c|c|c|c|c|}
\hline & & $\begin{array}{c}\mathrm{HR} \\
(\mathrm{bpm})\end{array}$ & $\begin{array}{l}\mathrm{RR} \\
(\mathrm{ms})\end{array}$ & $\begin{array}{l}\text { MxDMn } \\
(\mathrm{ms})\end{array}$ & $\begin{array}{l}\text { RMSSD } \\
(\mathrm{ms})\end{array}$ & $\begin{array}{c}\mathrm{Amo} 50 \% / 50 \\
(\mathrm{~ms})\end{array}$ & $\begin{array}{c}\mathrm{Si} \\
\text { (conventiona } \\
1 \text { units) }\end{array}$ \\
\hline \multirow{4}{*}{ 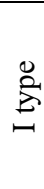 } & \multirow{2}{*}{ EG } & $90.4 *$ & $664.8 *$ & $249.9 *$ & 37.1 & $47.2 *$ & $139.3^{*}$ \\
\hline & & \pm 1.2 & \pm 21.6 & \pm 12.4 & \pm 1.8 & \pm 1.2 & \pm 12.4 \\
\hline & \multirow{2}{*}{ CG } & 92.1 & 642.35 & 221.1 & 34.8 & 52.8 & 153.5 \\
\hline & & 0.9 & 23.2 & 11.4 & 0.9 & 1.8 & 10.8 \\
\hline \multirow{4}{*}{ 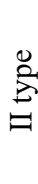 } & \multirow{2}{*}{ EG } & $91.8^{*}$ & 629.4* & 225.6 & $32.8^{*}$ & $63.1 * *$ & $160.2 *$ \\
\hline & & 1.04 & 11.5 & 7.9 & 1.1 & 1.2 & 11,3 \\
\hline & \multirow{2}{*}{ CG } & 93.42 & 611.1 & 212.5 & 27 & 67.3 & 233 \\
\hline & & \pm 0.75 & \pm 22.1 & \pm 9.1 & \pm 1.3 & \pm 0.7 & \pm 53.4 \\
\hline \multirow{4}{*}{$\stackrel{\text { ڤ }}{\stackrel{D}{\Xi}}$} & \multirow{2}{*}{ EG } & $83.4 *$ & $803.9^{*}$ & $338.9^{*}$ & $64.3 *$ & $30.9 * *$ & $64.6^{*}$ \\
\hline & & \pm 1.4 & \pm 24.2 & \pm 13.3 & \pm 1.9 & \pm 0.9 & \pm 2.2 \\
\hline & \multirow{2}{*}{ CG } & 86.9 & 766.6 & 313.8 & 61.9 & 33.4 & 69.3 \\
\hline & & \pm 0.6 & \pm 6.8 & \pm 14.1 & \pm 1.3 & \pm 0.75 & \pm 2.5 \\
\hline \multirow{4}{*}{ 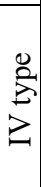 } & \multirow{2}{*}{ EG } & $68.1^{*}$ & $868.2 *$ & $527.2 *$ & $120.4 *$ & 22.4 & 14.9 \\
\hline & & \pm 1.5 & \pm 9.5 & \pm 14.04 & \pm 6.9 & \pm 0.7 & \pm 1.9 \\
\hline & \multirow{2}{*}{ CG } & 75.4 & 838.6 & 481.9 & 123.7 & 18.2 & 13.1 \\
\hline & & \pm 1.5 & \pm 11.08 & \pm 9.8 & \pm 8.66 & \pm 0.8 & \pm 1.16 \\
\hline
\end{tabular}

Note: $*$ - $\mathrm{p}<0.05, * *-\mathrm{p}<0.01$

Under the influence of a specialized load aimed at students in the EG, there was an improvement in heart rate variability indicators, a transition to a stable functional state of the cardiovascular system, body systems work in a normal mode, the state of regulatory systems is characterized by optimal activity, and refer to the physiological norm in the EG (Table 2).

In the $\mathrm{CG}$, at the end of the experiment, the overstrain of the regulatory systems remains, the state of the students belongs to the 5th functional class, which is characterized by the state of the body between the norm and the pathology. Prolonged overstrain can be the cause of the initial forms of various somatic diseases, depending on the predisposition $[7,11]$.

The received results in the EG show the need to correct physical activity with the help of special types of physical activity and exercises at physical culture lessons. Without the use of corrective and restorative motional influences at the lessons of physical culture in the $\mathrm{CG}$, the regulatory systems of the body of students are in the recovery mode, children of the second childhood need rest, and they are contraindicated in significant loads. The state of the regulatory systems is characterized by the prenozological state (Table 2). 
Table 2. Assessment of the functional state and regulatory systems of students at the start and at the end in the EG and CG study

\begin{tabular}{|l|c|c|c|c|}
\hline Estimation of the regulatory systems activity & $\begin{array}{l}\text { EG } \\
\text { start }\end{array}$ & $\begin{array}{c}\text { CG } \\
\text { start }\end{array}$ & $\begin{array}{c}\text { EG } \\
\text { end }\end{array}$ & $\begin{array}{c}\text { CG } \\
\text { end }\end{array}$ \\
\hline $\begin{array}{l}\text { Functional maintenance level: } \\
\text { Marked bradycardia (-2) - Moderate bradicardia (-1) - } \\
\text { Nomocardia (0) }\end{array}$ & -2 & -1 & 0 & -1 \\
\hline $\begin{array}{l}\text { Regulation stability: } \\
\text { Marked heart variability increase-arrhythmia (-2) - } \\
\text { Moderate heart variability increase (-1) - Normal heart } \\
\text { rhythm stability (0) }\end{array}$ & -2 & -1 & 0 & 0 \\
\hline $\begin{array}{l}\text { Vegetative homeostasis: } \\
\text { Marked predominance of parasympathetic system (-2) - } \\
\text { Moderate predominance of parasympathetic system (1) - } \\
\text { Normal vegetative balance (0) }\end{array}$ & -2 & -1 & 0 & 0 \\
\hline $\begin{array}{l}\text { Activity of sympathetic cardiovascular centre: } \\
\text { Marker vascular centre activity decrease (-2) - Normal } \\
\text { activity of vascular centre (0) }\end{array}$ & 0 & -2 & 0 & -2 \\
\hline $\begin{array}{l}\text { Centralization of control rate: } \\
\text { Marker of decrease of activity of central regulations levels } \\
\text { (-2) - Normal activity of central regulations levels (0) - } \\
\text { High activity of central regulations levels (1) }\end{array}$ & 0 & -2 & 1 & -2 \\
\hline $\begin{array}{l}\text { Functional overload: } \\
\text { Prenosological state (4-7) - Physiological norm (1-3) }\end{array}$ & 6 & 7 & 1 & 5 \\
\hline
\end{tabular}

Considering the indices of the distribution density of cardio intervals in the EG and CG on the graph, we observe that the variation range of the students from the EG increases and the mode amplitude decreases. In the CG, the indicators remain at the same level, the variation range decreases and the mode amplitude increases.

Thus, the use of correctional and restorative modes of physical activity during a physical culture lesson contributes to the improvement of the state of the functional systems of the body of students, the improvement of functional indicators in the EG, in contrast to the $\mathrm{CG}$, thereby preventing the breakdown of physiological adaptation under the influence of educational and physical loads.

In addition, students have different sex-age characteristics of the development of the cardiovascular system and the body as whole, different reactions of regulatory systems to the training load is realized, therefore, it is necessary to differentiate HRV changes from the standpoint of gender differences.

\section{Discussion}

Physical fitness is one of the conditions for personality development. Sport in combination with similar means of education, sport contributes to the all-round development of the student. Sport not only develops the physical body, but also affects the moral qualities, behavioural and volitional, cognitive and emotional spheres. Exercise affects not only a single organ, but the entire body as a whole through the nervous system. Therefore, even with minor physical exertion, a person immediately notes the changes that occur in many organs and systems. In the activity of the cardiovascular system, a general reaction to exercise: the pulse begins to accelerate (by 40-50\%) and the difference between systolic and diastolic blood pressure increases. As noted by scientists, such an increase in the amplitude of blood pressure may indicate an increase in the stroke volume of the heart. That is, the amount of blood that the heart can throw out in one contraction. Consequently, the minute blood volume also begins to increase. Organs and tissues receive more oxygen- 
enriched blood per unit of time, which improves their function and nutrition. Systematic physical exercise affects noticeable changes in metabolism, improves healthy appetite and gastrointestinal function. Also, under the influence of prolonged use of physiotherapy exercises, intestinal acidity is normalized. It is worth noting that systematic physical education has a positive effect on the human body [12-13]. Students who are systematically engaged in physical education are more active. They develop a certain daily routine; develop socially oriented attitudes and a higher vitality. This category of students often shows more responsibility, a sense of duty, conscientiousness in their studies, and selfdiscipline when answering. They interact more successfully in work that requires systematic stress on the body; it is easier for them to self-control. All this indicates a significant positive influence of systematic sports in the process of studying at the university. We also note that sport is an effective means of physical education. Rethinking the role of physical culture among young people, proceeding directly from the interests of a particular individual, would contribute to a tangible increase in the health benefits of physical education [14-15].

An individual approach is of particular importance, as even in groups with homogeneous states and disorders, the functional capabilities of each student are different. But the individual approach does not exclude the general properties inherent in all students. Therefore, it should be considered as an integral part of the pedagogical process. A differentiated approach to physical education of students of different groups and types is not limited only to volume and intensity, i.e. to the quantitative characteristics of the physical activity used [16-17]. The relationship between the dynamic structure of the motor act and the qualitative features of the reactions of the cardio respiratory system should be taken into account in the use of physical exercises of various orientations in the ecological and physical education of students. Taking into account the morphological and functional characteristics and disorders in the body of students, special exercises can be selected that can provide a reduced load for an organ weakened by a pathological process with sufficiently high loads for other functional systems [18].

\section{Conclusion}

The ecological culture of education is just beginning to take shape. in it, ergonomic and medical aspects play an important role, associated with compliance with the requirements for organizing educational work that does not harm the body. When organizing educational work, it is important to take into account both the gender (sex) and age characteristics of students and their individual characteristics, including the health group, sports or physical labor, the general level of bodily-physical culture, etc.

\section{References}

1. H. A. Aghajanyan, P. M. Baevskiy, A. P. Bersenev, Adaptation problems and the doctrine of health (Moscow: Publishing house of the Peoples' Friendship University of Russia, 2006)

2. V. Yu. Albitskiy, A. A. Modestov, E.V. Antonova, Russian Pediatric J., 4, 4-7 (2009)

3. P. M. Baevskiy, Bulletin of arrhythmology, 24, 65-86 (2001)

4. S. P. Arshinnik, V.I. Tkhorev, Physical culture. Sport. Tourism. Motor recreation 1, 3, 12-5 (2016)

5. D. N. Gavrilov, Medico-biological and pedagogical foundations of adaptation, sports activity and a healthy lifestyle: a collection of scientific articles of the III All-Russia. 
correspondence scientific-practical conf. with int. participation: in 2 volumes. 1, 24145, ed. G. V. Bugaeva, I. E. Popova (Voronezh: IPC "Scientific book", 2014)

6. N. I. Shlyk, E. N. Sapozhnikov, I congress of physiologists of the CIS: scientific works Vol 1, ed. R. I. Sepiashvili, 408 (Moscow: Medicine and health Publ, 2005)

7. N. I. Shlyk, E. N. Sapozhnikov, Russian J. of Physiology. THEM. Sechenov: theses of reports, 90, 8(2), 417 (2004)

8. P. M. Baevskiy, G. G. Ivanov, New methods of electrocardiography, 473-96 (Moscow: Technosphere, 2007)

9. P. M. Baevskiy, G. G. Ivanov, Ultrasound and functional diagnostics, 3, 108- 27 (2001)

10. P. M. Baevskiy, A. P. Bersenev, Assessment of the body's adaptive capabilities and the risk of developing diseases, 232 (Moscow: Medicine Publ., 1997)

11. R. A. Arynova, T. M. Shepilo, M. M. Malik, Youth of Siberia - the science of Russia international scientific and practical conference. Siberian Institute of Business, Management and Psychology, 29-32 (Krasnoyarsk: Editorial and Publishing Center of the Siberian Institute of Business, Management and Psychology, 2017)

12. D. Chadha, Researach in Science Education, 04, 1 (2020) https://doi.org/10.1007/s11165-020-09972-4

13. R. B. King, I. S. Caleon, Child Indicators Research, 14, 341-67 (2021) https://doi.org/10.1007/s12187-020-09757-1

14. N. A. Yuganova, D. A. Korshunov, M. A. Ikonnikova The Russian J. of physical education and sport, 12, 4, 83-89 (2017) https://doi.org/10/14526/04_2017_269.

15. V. B. Salakhova, E. R. Khairullina, D. R. Belinskaya et al. 2020 Eurasian J. of Biosciences, 14, 2, 5063-70 (2020)

16. V. V. Gorelik, Sports Medicine, 2, 5 (2015)

17. V. V. Gorelik, S. N. Filippova, V. S. Belyaev, B. N. Chumakov, J. of Human Sport and Exercise, 3, 4, 894-906 (2018)

18. V. Gorelik, V. Vlasov, V. Makarova, J. of Physical Education and Sport, 18, 4, 2397403 (2018) 